\title{
Partial Sublimis Transfer
}

\section{Suggestion for New Type of Leprosy Hand Surgery}

\author{
TH. WYSS, M.D. \\ Sacred Heart Leprosy Hospital, Sakkotai P.O., via Kumbakonam, Madras State, India
}

From 1959 to 1966 research was made at the Sacred Heart Leprosy Hospital, Kumbakonam, Madras State, India, in order to find more suitable methods for claw hand rehabilitation.

Long term observation of the old sublimis transfer operations have shown the opposite deformity after 1 or 2 years. These overcorrection deformities are mainly due to the complete removal of the sublimis tendon and are prohibitive in the case of fingers with normal mobility. In order to prevent these sublimis removal deformities ingenious procedures have been developed by P. Brand, Vellore. The sublimis tendon is left in situ and a prolonged tendon of the extensor carpi radialis brevis is connected with the paralysed finger extensors.

The new suggestion: Why transfer a whole sublimis, where half is enough? One half of the sublimis tendon is used for the extension of the finger-joints of 2 adjacent (=neighbouring)

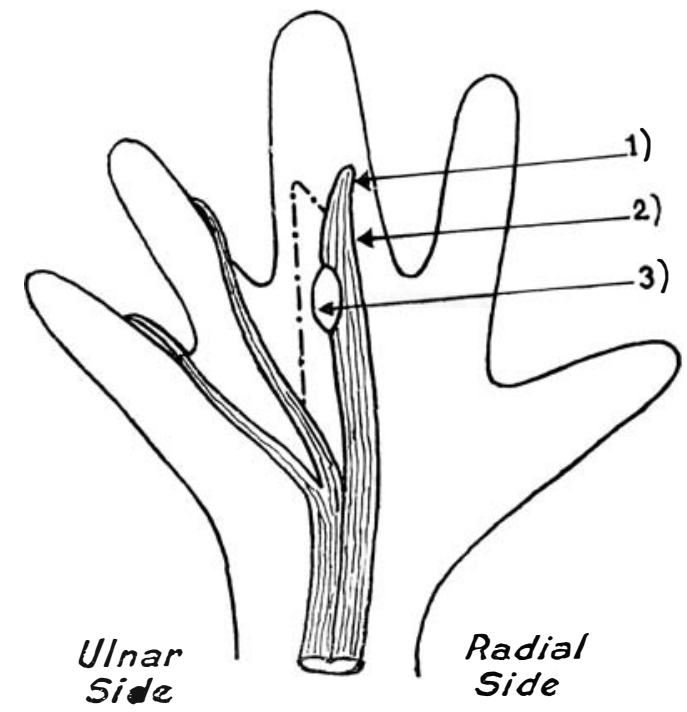

1. Insertion of sublimis tendon.

2. Chiasma-part of sublimis tendon.

3. Profundus canal. fingers. This partial sublimis transfer cannot work on one and the same finger, because the remaining half will flex the finger-joints, whereas the transferred half would extend them and interfere.

Example: Clawing of ring and little finger (very common in leprosy). Ulnar half of long finger sublimis is split off, pulled back into the palm and divided in 2 tails. Each tail is passed through the lumbrical canal and stitched into the extensor expansions of ring and little finger, giving extension to the finger-joints. Radial half of long finger remains in situ, preventing sublimis removal deformity.

Detailed description of the PARTIAL removal of the sublimis tendon.

(Without damaging the flexor sheet proximal prof undus canal !)

1. Terminal anaesthesia at the base of long finger. Bloodless field by tiny rubber-band, distal MP joint*. Ventrolateral incision at level of proximal finger joint. Flexor sheet opened. In maximal flexion of MP joint* the ulnar tail of the sublimis tendon is pulled out by dental hook. Ulnar tail is held by assistant. Chiasma-part of sublimis tendon pulled out and lifted, so that the 'bottom' of the chiasma and the vinculum is visible. Now the vinculum is cut. Complete detaching of all the vincula is essential, otherwise the sublimis tendon cannot be pulled out.

2. In maximal flexion of wrist and MP joint*: the whole sublimis tendon is pulled out, until the profundus canal is visible. Under control of the eye the chiasma part of the sublimis tendon is split from the prof undus canal towards the insertion. Radial and ulnar tails of the sublimis tendon are thus separated.

3. Only now ulnar half is detached from the insertion. Undetached radial half is pulled out and fixed by assistant. Loose ulnar tail is caught by mosquito forceps. By simple pulling on the mosquito forceps the sublinis tendon is rent in two tails, backwards from profundus canal. Extreme flexion of wrist and MP joint* will make this splitting procedure easy and will give a nice long rent far back into the palm. Flexor sheet proximal profundus canal remains untouched.

* Metacarpo-Phalangeal joint. 


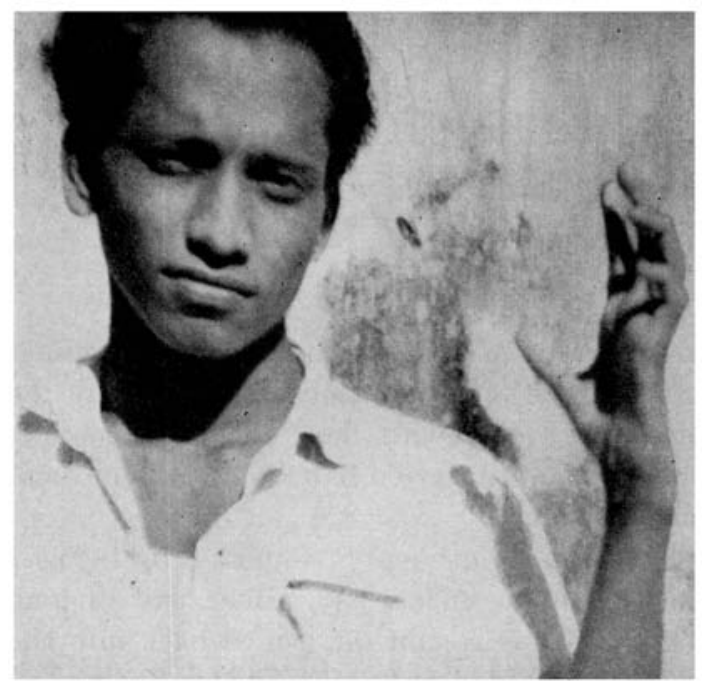

'Two-finger deformity.' Clawing of ring and little finger.

4. Under local anaesthesia incision in the distal fold of the palm. Sublimis tendon is taken out from the flexor sheet. The rent in the tendon is identified (extreme flexion of the finger-joints very helpful!). Now the loose ulnar-half can be pulled out separately into the palm. A second time the ulnar half is torn back and cut into two tails. Each tail is passed by tunnel forceps through the lumbrical canal and stitched into the extensor expansions of ring and little finger. The stitching must be made under some tension, as there will be no helpful retraction as in case of complete sublimis removal.

Before doing the partial sublimis transfer we must make sure that there is sufficient power in the sublimis tendon. Failures are often due to powerless long finger sublimis, sometimes paralysed in violation of the rule that leprosy will damage only the small hand muscles and spare the forearm muscles.

Four finger disabilities can be operated in the same way: Long finger sublimis providing its ulnar half for index and ring finger, Index finger sublimis supplying ulnar half for long and little finger. Ring finger sublimis is spared for thumb reconstruction. Post-operative fixation is made in extreme flexion of the wrist joint, by special aluminium splints, avoiding plaster. For prevention of adhesions, early beginning of physiotherapy is essential. Failure has occurred, but was mainly due to powerless sublimis tendon or to insufficient tension in tying the sublimis tendon to the extensor expansion.

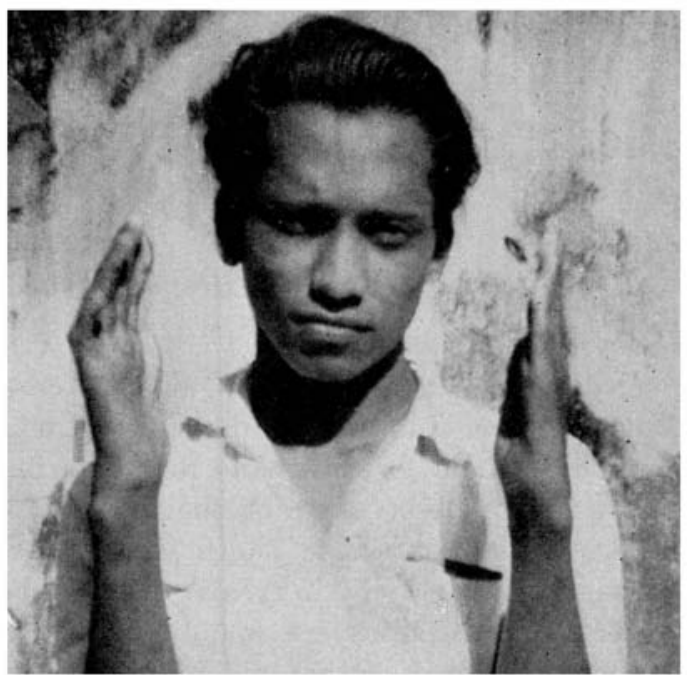

One year after the partial transfer of long finger sublimis.

A batch of 20 people have been operated on by the new method at the Sacred Heart Leprosy Hospital, Kumbakonam. Seventeen of them show satisfactory correction of their claw hand disability without a shade of over-correction. Contractures of the finger joints are always a strong contraindication for this type of transfer.

\section{SUMMARY}

A method of claw hand operation, using partial sublimis transfer, is described. The new procedure is especially convenient for reconstruction of isolated clawing of ring and little finger, very common in leprosy.

\section{ACKNOWLEDGEMENTS}

I am very grateful to Dr. N. Zwicky, M.D., to Mr. C. Pereira, as well as to the management of Sacred Heart Leprosy Hospital, for the assistance and encouragement in the production of this article.

\section{REFER ENCES}

BRAND, P. w. (1958). Leprosy in Theory and Practice. COCHRANE

BRAND, P. w. (1959). Internat. J. Lep., 27, $1-7$.

BRAND, P. W. (1963). Rehab. Lit., 21, 238-245.

Cochrane, R. G. Leprosy in Theory and Practice,

Chap. on Reconstr. Surgery.

FRITSCHI, E. P. (1958). Leprosy in India. 